\title{
Trade-off Between the Sampling Rate and the Data Accuracy
}

\author{
Chun Zhang and Xue Liu
}

\begin{abstract}
In this paper, we investigate the trade-off between the sampling rate and the data accuracy as a simplified model for resource allocation between different components of a networked control system. The problem is set up as the classical Linear Quadratic Gaussian (LQG) control problem of a sampled-data system over a discrete-time communication channel. We show that the LQG cost can be divided into the control cost, the communication cost, and the sampling cost. Through numerical examples, we demonstrate that the controller prefers to have more frequent communication with the plant, even if the information is crude instead of to obtain a piece of more precise information with a long delay. The main contribution of this paper is to provide a method to evaluate the time value of information in a control system.
\end{abstract}

\section{INTRODUCTION AND RELATED WORKS}

In networked control systems, resource sharing between components of the system brings in the fairness issue of resource allocation. In this paper, we consider the allocation problem of the limited communication resource and as a start, we consider the impact of the limited average throughput (data rate) on the performance of a centralized system. By choosing an appropriate cost function, the mechanism developed here can be generalized to distributed systems with multi-subsystems.

The problem is setup as the classical LQG problem of a sampled-data system over a discrete-time additive white Gaussian noise (AWGN) channel. We investigate the tradeoff between the sampling rate and the data accuracy for a given average throughput, which is the figure of merit in stabilizing such a control system, [15]. The sampling rate indicates how frequently the system has to access the communication resource and the data accuracy is an indicator of the required channel capacity at each transmission.

The sampled-data problem has been well studied. Book [4] provides a through survey of various aspects of sampled-data control problems. Reference [2] deals with the $\mathcal{H}_{\infty}$ problem of a sampled-data system while reference [3] solves the $\mathcal{H}_{2}$ problem. Paper [12], [14] consider the effect of the sampling rate on the LQ cost using a more computer science approach.

The control over communication channel problem has been a major research attraction for the past two decades. Since paper [5], various authors address the stabilization problem of communication constrained control systems, see [10], [13], [16], [6], [8], [19], [20] and reference therein. However, there are only a few works on performance problems of such systems. Tatikonda and Mitter considered

Chun Zhang is with the Department of Electrical and Computer Engineering, University of Illinois, Urbana, IL 61801, USA czhang4 @uiuc. edu

Xue Liu is with the School of Computer Science, McGill University, Montreal, Quebec, Canada, H3A 2A7 xueliuecs.mcgill.ca the LQG control problem of a discrete-time Linear TimeInvariant (LTI) system over a communication channel in [17], upon which our result is built. Paper [9] considers the Linear Quadratic Regulation (LQR) problem of a scalar discrete-time LTI system with static quantizers under highrate assumption. Paper [11] considers the state regulation problem of a sampled-data system under a similar setup as Ours. References [7], [18] also investigate some performance problems with other specific performance criteria.

In most of the previous works on controls with limited communication, discrete-time LTI systems are assumed. The communication channel is also assumed to be a compatible discrete-time channel, where the channel capacity is measured in bits per sample (bits per channel use).

However in real life applications, a control system works with continuous-time plants. Thus sampling is necessary for implementing digital control. The network resource is usually valued in terms of b.p.s. (bits per second) as the average throughput, which can be considered as the product of bits per sample and samples per second. The former measures the channel capacity of the equivalent discrete-time channel, in other words, per sample channel capacity, which determines the data accuracy of the sample. The later is exactly the sampling rate, which measures how frequently the system has to access the communication resource. Thus in order to properly evaluate the performance, one must explicitly consider the channel accessing frequency together with the available channel capacity at each transmission. Therefore, a more appropriate measure of the communication constraint is the average throughput instead of the channel capacity.

Given fixed average throughput, one can trade off between the sampling rate and the data accuracy. In the stabilization problem, these changes do not make any difference since the average throughput is the unique measure of the required information rate, [15]. However, this is generally not true in performance problems. In this paper, we show that the LQG cost of the sampled-data system can be decomposed into three parts: the control cost, the communication cost, and the sampling cost. The total cost is inversely related to both the sampling rate and the data accuracy. Increasing either of them decreases the cost. So the natural question to ask is what is the optimal trade-off between the sampling rate and the data accuracy given fixed network throughput?

We analytically and quantitatively investigate this problem, and discuss the trade-off between these two factors. Our main contribution is to show that in order to minimize the LQG cost, sampling the system faster, aka, communicating more frequently is more effective than sending a more accurate information with a long delay given fixed average rate. This 
is amount to say that information itself has a time value.

The rest of the paper is organized as follows. The problem is set up in Section II. Section III derives the cost function analytically. In section IV, we use numerical examples to show the trade-off between the sampling rate and the data accuracy along with evaluations. The conclusions are given in Section V.

\section{PROBLEM FORMULATION}

The system under consideration is illustrated in Fig. 1.

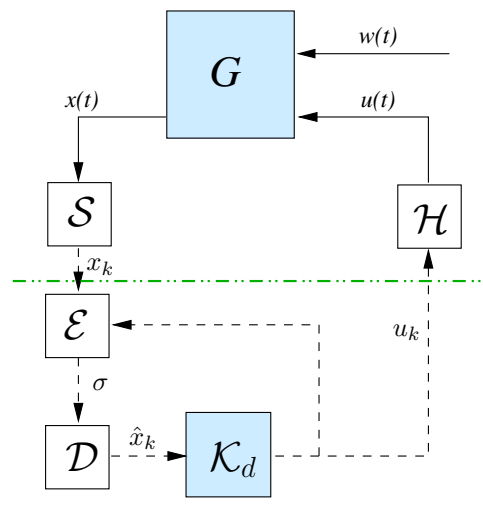

Fig. 1. Sampled-Data System with Communication Constraints

The plant $G$ is a continuous-time LTI system with the following state-space representation

$$
\dot{x}(t)=A x(t)+B u(t)+G w(t)
$$

where state $x \in \mathbb{R}^{n}$, control $u \in \mathbb{R}^{u}$. The external disturbance $w \in \mathbb{R}^{w}$ is an i.i.d. zero mean Gaussian process with auto-correlation function $K_{w}\left(t_{1}, t_{2}\right)=\Gamma \delta\left(t_{1}-t_{2}\right)$. Matrices $A, B$ and $G$ are of compatible dimensions; And when the system is scalar, we use lower case letters $a, b$ and $g$ to denote them. For convenience, we assume state feedback in our setup.

The sampler $\mathcal{S}$ and the zero-order hold $\mathcal{H}$ are assumed to have perfect synchronization. The sampling period is denoted as $h$, with $f=\frac{1}{h}$ as the sampling rate. The sampler and the hold work as follows

$$
\begin{aligned}
& x_{k}=\mathcal{S} x(t) \text { s.t. } x_{k}=x(k h), \text { for } t \leq k h<(k+1) h \\
& u(t)=\mathcal{H} u_{k} \text { s.t. } u(t) \equiv u_{k}, \text { for } t \leq k h<(k+1) h
\end{aligned}
$$

It is worth mentioning that the system is partitioned into a continuous-time part and a discrete-time part after sampling. We can assume that the discrete-time part (which is below the dash-dotted line in Fig. 1) only operates on time $t=k h$.

The encoder $\mathcal{E}$ at time $t=k h$ is a map $\mathcal{E}^{k}: \mathbb{R}^{n} \times$ $\Sigma^{[0, k-1]} \times \mathbb{R}^{u k}$ to $\Sigma$, taking values $\left(x_{k}, \sigma^{[0, k-1]}, u^{[0, k-1]}\right) \mapsto$ $\sigma(k)$, where $\Sigma$ is the codeword space and $\Sigma^{[0, k-1]}$ denotes the union of all the past codeword spaces. Notation $\sigma^{[0, k-1]}$ is used to denote all the past codewords and $u^{[0, k-1]}$ is used to denote all the past controls. The encoder knows the decoding policy $\mathcal{D}$ but not the control policy $\mathcal{C}$.

The decoder $\mathcal{D}$ at time $t=k h$ is a map $\mathcal{D}^{k}: \Sigma^{[0, k]} \times \mathbb{R}^{u k}$ to $\mathbb{R}^{n}$, taking values $\left(\sigma^{[0, k]}, u^{[0, k-1]}\right) \mapsto \hat{x}_{k}$. The decoder knows the encoding policy $\mathcal{E}$ but not the control policy $\mathcal{C}$. The output of the decoder is an estimate of the state $x(k h)$.

The discrete-time controller $\mathcal{K}_{d}$ at time $t=k h$ is a map $\mathcal{C}^{k}: \mathbb{R}^{n(k+1)}$ to $\mathbb{R}^{u}$ taking values $\hat{x}^{[0, k]} \mapsto u_{k}$. The control causally depends on the decoder's outputs.

The channel connecting the encoder and decoder is assumed to be a discrete-time AWGN channel with capacity $R$ bits/ch use (per sample channel capacity).

The cost function is the classical LQG cost given as

$$
J=\limsup _{N \rightarrow \infty} \frac{1}{N} \mathbb{E}\left[\int_{0}^{N h} x^{\prime}(t) \tilde{Q} x(t)+u^{\prime}(t) \tilde{S} u(t) d t\right]
$$

where $N$ is a positive integer and $\mathbb{E}$ is used to denote the expectation. The transpose of $x(t)$ is denoted as $x^{\prime}(t)$.

Our goal is to solve the following optimization problem:

$$
\begin{aligned}
& \min J \\
& \text { subject to } \operatorname{Eqn}(1) \text { and } f R=c \text { b.p.s.. }
\end{aligned}
$$

where $c$ is the given fixed average throughput.

In the next section, we analytically evaluate this problem and in section IV, we show some interesting trade-offs between $f$ and $R$ through numerical examples.

\section{EVALUATION OF THE COST}

In this section, we show how to break the cost $J$ into three parts, which can be then evaluated separately. The detailed mathematical derivations are omitted due to page limitation and are available upon request.

We first consider sampling a system with no communication constraints. The LQG cost (2) can be divided into the LQG cost of an equivalent discrete-time LTI system and an additional cost which quantifies the sampling effects.

Then we consider the communication constraints, the LQG cost of the equivalent discrete-time LTI system can be further decomposed into two parts: the control cost and the communication cost. Thus we have three costs in total.

\section{A. Effects of Sampling}

Let us for now assume the communication channel is perfect, that is, the sampled output $x(k h)$ can be observed by the controller $\mathcal{K}_{d}$ without any distortion. With the sampler $\mathcal{S}$ and the hold $\mathcal{H}$, the original system (1) is transformed to the following equivalent discrete-time LTI system

$$
x_{k+1}=A_{d} x_{k}+B_{d} u_{k}+v_{k}
$$

where

$$
\begin{aligned}
x_{k} & =x(k h) \\
v_{k} & =\int_{k h}^{(k+1) h} e^{A((k+1) h-\tau)} G w(\tau) d \tau \\
A_{d} & =e^{A h} ; \quad \mathbb{R}^{n} \rightarrow \mathbb{R}^{n} \\
B_{d} & =\int_{0}^{h} e^{A \tau} d \tau B ; \quad \mathbb{R}^{u} \rightarrow \mathbb{R}^{n}
\end{aligned}
$$

We have the following results on the sampled system 
Lemma 1: The disturbance $v_{k}$ is a zero mean i.i.d. Gaussian process with variance

$$
K_{v}=\int_{0}^{h} e^{A \tau} G \Gamma G^{\prime} e^{A^{\prime} \tau} d \tau
$$

Lemma 2: The cost function $J$ has the following expression after sampling

$$
\begin{aligned}
J= & \limsup _{N \rightarrow \infty} \frac{1}{N} \mathbb{E}\left[\sum_{k=0}^{N-1} x_{k}^{\prime} Q x_{k}+2 x_{k}^{\prime} M u_{k}+u_{k}^{\prime} S u_{k}\right] \\
& +\Delta(h)
\end{aligned}
$$

where

$$
\begin{aligned}
Q & =\int_{0}^{h} e^{A h} \tilde{Q} e^{A h} d t \quad ; \quad M=\int_{0}^{h} e^{A h} \tilde{Q} H(t) d t \\
S=\int_{0}^{h}(\tilde{S} & \left.+H^{\prime}(t) \tilde{Q} H(t)\right) d t ; H(t)=\int_{0}^{t} e^{A \tau} d \tau B \\
W(t) & =\operatorname{Trace}\left\{\int_{0}^{t} \tilde{Q}^{\frac{1}{2}} e^{A \tau} G \Gamma G^{\prime} e^{A^{\prime} \tau} \tilde{Q}^{\frac{1}{2}} d \tau\right\} \\
\Delta(h) & =\int_{0}^{h} W(t) d t
\end{aligned}
$$

and $\Gamma$ is the covariance matrix of $w(t)$ as defined in the previous section.

Now, the original LQG problem for the continuous-time system (1) is transformed to the equivalent discrete-time LQG problem given by (3) and (5). The cost function $J$ has a crossover term $2 x_{k}^{\prime} M u_{k}$ and an extra term $\Delta(h)$, which quantifies the sampling effects and it is independent of controls.

\section{B. Effects of Communication Constraints}

For a discrete-time Gaussian-Markov source, paper [17] solves the LQG problem (with no crossover terms in the cost function) over a capacity constrained channel. The sequential rate distortion framework is introduced and a separation of the total cost into a full knowledge cost and a sequential rate distortion cost is derived. Specifically, for a system

$$
z_{k+1}=F z_{k}+H f_{k}+e_{k}
$$

where $z_{k} \in \mathbb{R}^{n}$ is the state, $f_{k} \in \mathbb{R}^{f}$ is the control and $e_{k} \in \mathbb{R}^{n}$ is an i.i.d. Gaussian noise, matrices $F$ and $H$ are of compatible dimensions. The following LQG cost

$$
\hat{J}=\limsup _{N \rightarrow \infty} \frac{1}{N} \mathbb{E}\left[\sum_{k=0}^{N-1} z_{k}^{\prime} \hat{Q} z_{k}+f_{k}^{\prime} \hat{S} f_{k}\right]
$$

where again $N$ is a positive integer and $\hat{Q} \geq 0$ and $\hat{S}>0$ can be decomposed into two parts

$$
\operatorname{Trace}\left(\hat{P} \hat{K}_{e}\right)+\operatorname{Trace}\left(\left(F^{\prime} \hat{P} F-\hat{P}+\hat{Q}\right) \Theta\right)
$$

where $\hat{K}_{e}$ is the covariance matrix of $e_{k}$, and $\Theta$ is the covariance matrix of the error between $z_{k}$ and its estimate $\hat{z}_{k}$. The matrix $\hat{P}$ is the solution to the optimal LQG problem when full information is available (no communications constraints), i.e., $\hat{P}$ is the solution to the following Riccati equation

$$
\hat{P}=F^{\prime}\left(\hat{P}-\hat{P} H\left(H^{\prime} \hat{P} H+\hat{S}\right)^{-1} H^{\prime} \hat{P}\right) F+\hat{Q}
$$

By noticing that the equivalent discrete-time LTI system (3) is in the exact form of (6), and the cost (5) is also similar to (7), we have the following theorem by combining this result and that in Lemma 2

Theorem 1: By using the following certainty equivalent controller

$$
u_{k}^{*}=-\left(R+B_{d}^{\prime} P B_{d}\right)^{-1}\left(B_{d}^{\prime} P A_{d}+M\right) \hat{x}_{k}
$$

where $P$ is the solution to the following Riccati equation

$$
\begin{aligned}
P= & \left(A_{d}^{\prime} P A_{d}+Q\right)-\left(B_{d}^{\prime} P A_{d}+M\right)^{\prime} \\
& \times\left(S+B_{d}^{\prime} P B_{d}\right)^{-1}\left(B_{d}^{\prime} P A_{d}+M\right)
\end{aligned}
$$

on system (1), the LQG cost (2) decomposes as follows

$J=\operatorname{Trace}\left(P K_{v}\right)+\operatorname{Trace}\left(\left(A_{d}^{\prime} P A_{d}-P+Q\right) \Lambda\right)+\Delta(h)$

where $\Lambda=\operatorname{Cov}\left(x_{k}-\hat{x}_{k}\right)$ is the covariance matrix of the estimation error over the channel.

Remark 1: Equation (9) says the cost $J$ decomposes into three parts: the first term is the control cost under full observation of the state $x(k h)$ and the second term characterizes the communication cost while the third term is a measure of the sampling cost. All costs depend on the sampling rate but only the second term depends on the channel capacity.

Paper [17] also shows that the estimation error is characterized by the sequential rate distortion. For a given data accuracy, the minimal distortion/cost is achieved over a matching channel ${ }^{\text {I }}$. For a Gaussian-Markov source as the one in (3), the matching channel is an AWGN channel with memory, which can be realized with an AWGN channel with noiseless feedback. Since our encoder knows the decoder's output, it can be regarded as a noiseless feedback. Thus with the given encoder, decoder and controller triple, the optimal cost can be computed explicitly for a scalar system. For a higher dimensional system, the cost can only be computed in low distortion (high rate) region. Readers are referred to [17] for detailed discussion.

\section{NUMERICAL EXAMPLES}

In this section, numerical examples are employed to show the interesting trade-off between the sampling rate $f$ (samples/sec) and the channel capacity $R$ (bits/sample). We first derive the exact cost function of a scalar system and then evaluate the cost under various parameter combinations to show how the system performance is affected by the average throughput $c$, the channel capacity $R$ (data accuracy), the sampling rate $f$, and plant parameters $A, B$, and $G$.

\section{A. Cost of Scalar Systems}

From now on, we assume system (1) is a scalar GaussianMarkov process, then following the derivations in previous

\footnotetext{
TProbability distribution of input-output of the channel matches the source, see [17] for more information.
} 
sections and the result in [17], the cost can be decomposed as in (9), which can be further evaluated as

$$
p k_{v}+\frac{k_{v}\left(a_{d}^{2} p-p+q\right)}{2^{2 R}-a_{d}^{2}}+\Delta(h)
$$

where lower case letters are used to denote scalar variables.

Clearly, when the channel capacity $R \leq \log _{2}\left|a_{d}\right|$ bits per sample, the cost is infinity, our goal is to evaluate the cost function (10) given $R>\log _{2}\left|a_{d}\right|$ and $R / h=c$ b.p.s.

It is straightforward to check that $R>\log _{2}\left|a_{d}\right|$ bits per sample is equivalent to $c=R f=R / h>|a| \log _{2} e$ b.p.s., which is the unique measure of the minimal average throughput required to stabilize the system. For multi-dimensional system, $c>\sum_{|\lambda(A)| \geq 1}|\lambda(A)| \log _{2} e$ is the information lower bound for stabilization. This is consistent with results in [15].

Analytical evaluation of the cost function (10) is a formidable task due to all the integrations involved in the parameters. Instead we present some numerical examples to illustrate the trade-off.

We consider the following parameter combinations for system (1) in order to see how the performance is affected. Evaluations are given along with figures.

We set $\Gamma=1, \tilde{q}=2$, and $\tilde{s}=1$, and

\begin{tabular}{|r|r|r|r|}
\hline & $a$ & $b$ & $g$ \\
\hline I & 2 & 1 & 1 \\
\hline II & 4 & 1 & 1 \\
\hline III & 2 & 10 & 1 \\
\hline IV & 2 & 1 & 2 \\
\hline
\end{tabular}

For the ease of plotting figures, when $R \leq \log _{2}\left|a_{d}\right|$, the cost is set to -100 , when any cost $>300$, it is set to -50 . For 3-d figures below, $x$-axis denotes the sampling period $h$ instead of the rate $f=\frac{1}{h}, y$-axis represents the channel capacity $R, z$-axis is the cost. For 2 -d figures, $x$ axis represents the sampling period $h$ and $y$-axis is the cost. We consider the following parameter ranges: $0.01 \leq h \leq 1$ second and $0.01 \leq R<5$ bits per sample.

\section{B. Effects of the Throughput, the Sampling Rate and the Channel Capacity}

Fig. 2 is the total cost LQG $J$ (2) for scenario (I).

From this figure, we have the following observations:

1 ), The most important one is that the cost $J$ is very small when the sampling rate $f$ is very high (corresponding to small $x$-axis numbers). This is not hard to understand because when sampled fast, the system matrix $a_{d}$ is very close to 1 , thus only a little effort is required to stabilize the system. A channel with small capacity is sufficient, which results in a low communication cost. On the other hand, the variance of the lifted noise $v_{k}$ is small due to the small sampling period, thus the control cost is low. Similarly the sampling cost is also very low due to fast sampling, which can be easily observed from the expression of $\Delta(h)$.

2 ), There is a clear cut of minimal required average rate to stabilize the system, this is represented by the line on $x-y$ plane which is determined by the points with $z=-100$. This line can also be observed if we penalize high cost,

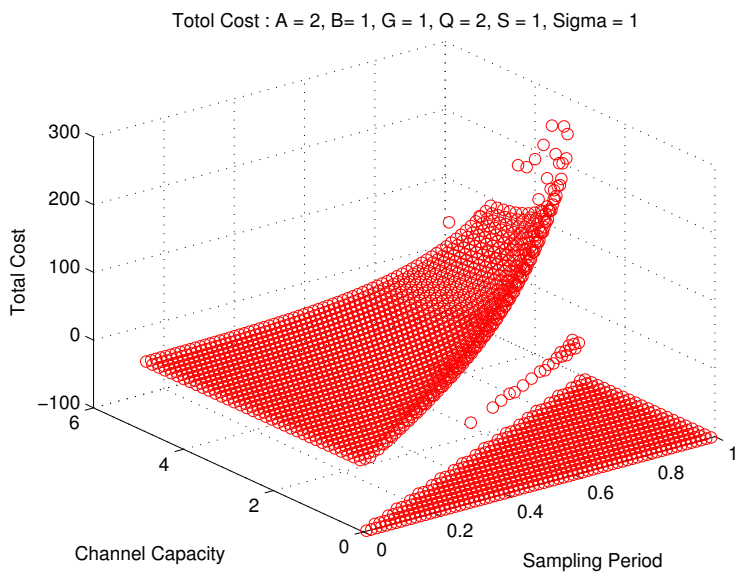

Fig. 2. Total Cost $J$ for Scenario (I)

say $J>500$, instead of checking the minimal required throughput. This is consistent with the minimal average throughput requirement derived earlier.

3 ), When the average throughput is just over the minimal requirement for stabilization, the overall LQG cost can still be high, this is shown by the -50 values in the figure, and the communication cost contributes to this explode since the channel capacity $R=c f$ is consequentially barely enough.

4), Increasing the channel capacity also helps to decrease the total cost, however, when the system is sampled very slowly, the control cost and sampling cost are dominant. Even with a large data rate, the decrease in the communication cost is not enough to offset the increase in the other two costs. Thus the total cost tends to get large regardless of the data accuracy. This is also illustrated in the next figure.

Fig. 3 shows how the separated three costs (red - control the middle layer, green - communication - the layer cutting through, and magenta - sampling - the lower layer) interacts with each other for parameter combination (I).

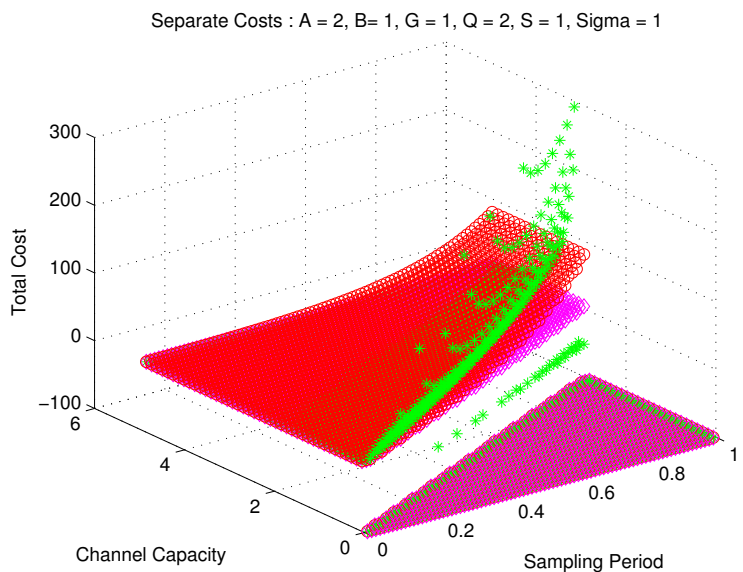

Fig. 3. Separate Costs for Scenario (I)

As we can see from the figure, the control cost and the 
sampling cost strictly increase with the sampling period $h$, and they are not related to the channel capacity. The communication cost is huge when the capacity is barely enough to maintain stability. But as the channel capacity gets higher, the communication cost drops very fast. On the other hand, lower sampling rate tends to incur higher communication cost, this is due to the larger system matrix $a_{d}=e^{a h}$ generated after sampling, which makes stabilization more demanding. When the per sample channel capacity is high, the control cost and the sampling cost dominate the communication cost. Thus, we conclude that the data should be sufficiently accurate but after some point, increasing the data accuracy is not very effective in lowering the total cost.

This phenomena was also observed in [17], where it shows that for a discrete-time LTI system over a communication channel, it is almost useless to increase the channel capacity beyond a certain point in order to reduce the LQG cost.

Fig. 4 shows the communication cost and the sampling cost for a given average throughput $c=6$ b.p.s., which is much higher than the minimal required stabilizing throughput, which is $2 \log _{2} e$ b.p.s.

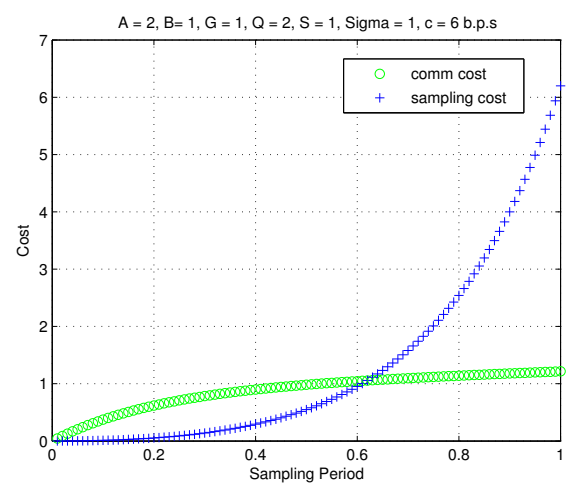

Fig. 4. Communication and Samping costs for Scenario (I)

We can see that the communication cost starts low and then increases a little bit but eventually decreases with the sampling period $h$. This is due to the fact that the per sample channel capacity $R$ is linearly related with the sampling period $h$ for a fixed $c$. However the cost does not linearly depend on either $R$ or $h$, thus its behavior is also nonlinear. The sampling cost, on the other hand, increases monotonically. Therefore, there is an optimal sampling rate for the total cost, especially when the channel capacity is low. This is also illustrated in Fig. 5, where the communication cost is substantially large. The control cost, which is not shown in this figure, also increases monotonically with $h$.

Fig. 5 is the communication cost given different average throughputs, namely, $c=2 \log _{2} e+0.2, c=2 \log _{2} e+0.5$, and $c=2 \log _{2} e+4$ bits per second (from high to low), where $2 \log _{2} e$ b.p.s. is the minimal required average throughput for stabilization. We observe that when the throughput is marginally enough, the communication cost is huge, but it decreases very fast as the throughput gets higher. Consider that $R=c h$, increase $c$ is effectively the same as increase
$R$ for a fixed sampling rate, thus initially increase the data accuracy is very effective in bringing down the communication cost. But when the data is sufficiently accurate, the communication cost is very low, then continuing to increase data accuracy is not economical. This is shown in the low cost region ( $y$ close to 0 ) in the figure.

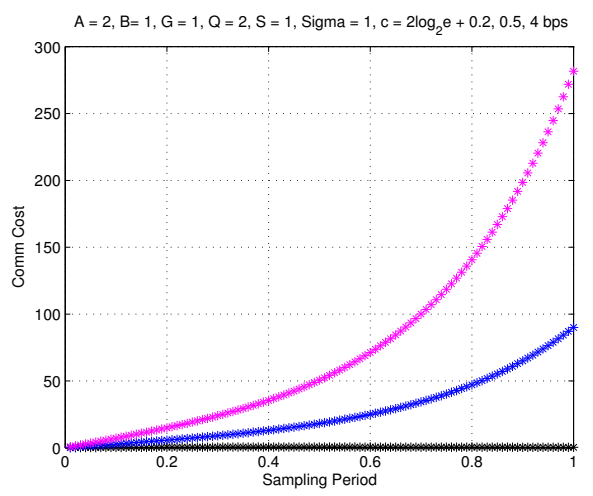

Fig. 5. Total Cost for Scenario (I) for Different b.p.s.

\section{Effects of Plant Parameters}

We now investigate how plant parameters affect the cost.

Fig. 6 shows how the parameter $a$ affects the total cost, the lower (red) layer is the cost for scenario (I) with $a=2$ (same as Fig. 2) and the upper (blue) layer is the cost for scenario (II) with $a=4$. From this figure we observe that,

1), Larger $a$ value does require higher average throughput for stabilization. Notice the blue line on the $x-y$ plane has a larger slope than the red line. This is equivalent to say that $R_{2} / h_{2}>R_{1} / h_{1}$, i.e., more b.p.s. required.

2), Larger $a$ value tends to generate higher cost for fixed $R$ and $h$ due to the increase in all three individual costs.

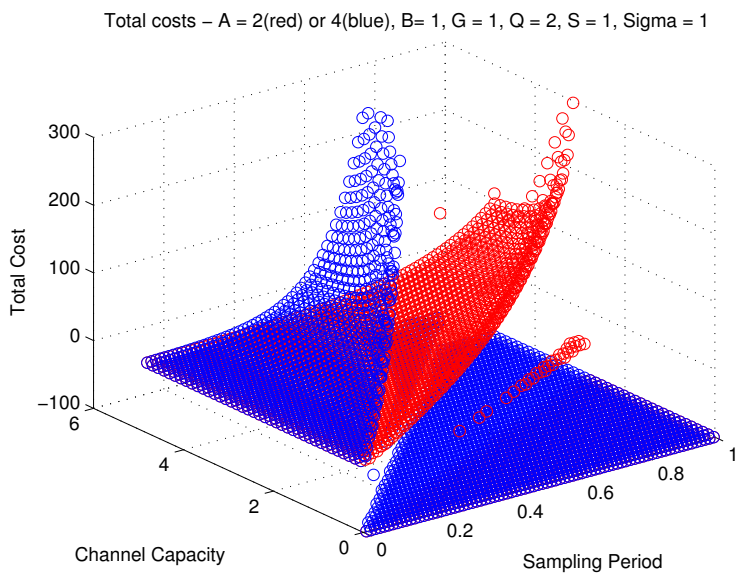

Fig. 6. Total Cost for Scenario (I) and (II)

Fig. 7 shows how the parameter $b$ affects the total cost, the upper (red) layer is the cost for scenario (I) with $b=1$ (same as Fig. 2), the lower (blue) layer is for scenario (III) 
with $b=10$. From this figure we can see that a larger $b$ value can reduce the cost, this is due to the smaller $p$ value computed. Also, it is worth mentioning that the stabilizing average throughput does not change under different $b$ values.

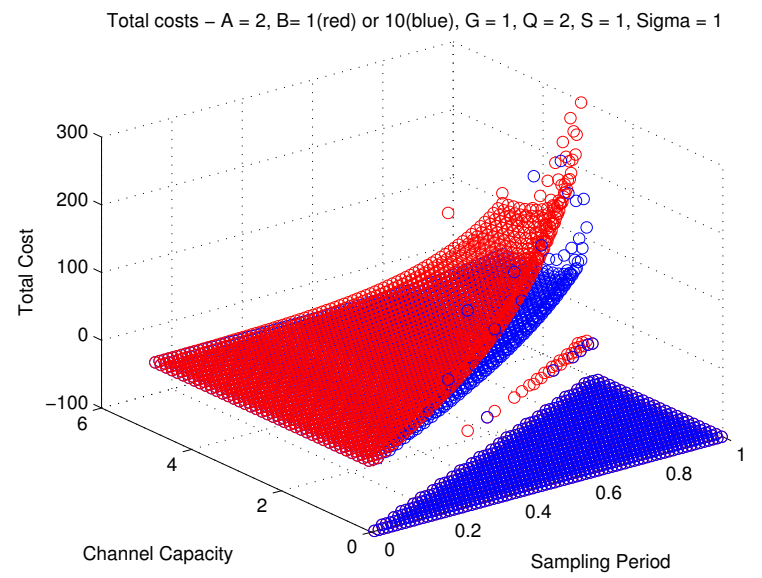

Fig. 7. Total Cost for Scenario (I) and (III)

Fig. 8 shows how the parameter $g$ affects the total cost, the lower (red) layer is the cost for scenario (I) with $g=1$ (same as Fig. 2), the upper (blue) layer is for scenario (IV) with $g=2$. It is not surprising that the required throughput for stabilization does not change but the cost increases due to a noisier process enters the system. The same result can be obtained by enlarging the covariance matrix $K_{w}$.

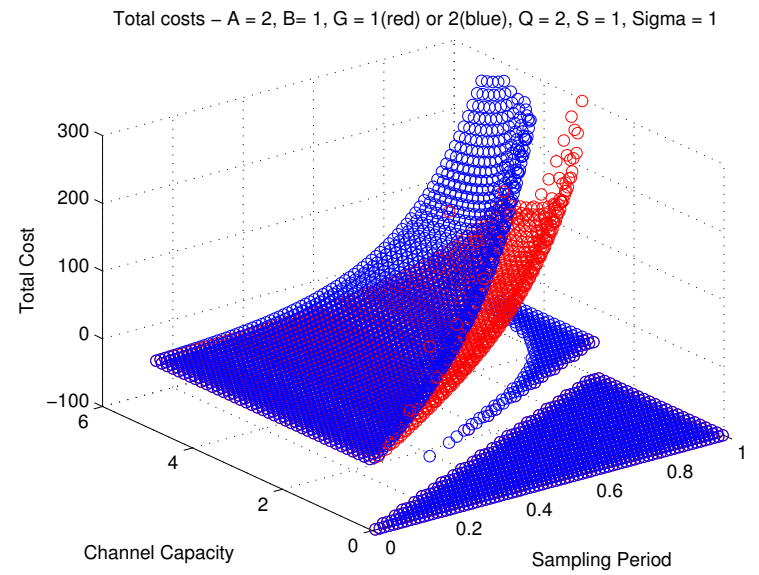

Fig. 8. Total Cost for Scenario (I) and (IV)

\section{CONCLUSIONS}

For control system over communication networks, how to allocate the communication resource fairly is an important issue. In this paper, we investigated the sample model of the LQG performance problem of a sampled-data system over a discrete-time capacity limited communication channel. Through numerical examples we demonstrate the trade-offs between the sampling rate and the data accuracy. We infer that given fixed network resources, in order to minimize the cost, the controller prefers to have more frequent communication with the plant than to obtain more accurate information with a long delay. The fundamental axiom in finance says "money has a time value", analogously we conclude that "information has a time value".

Our future work include extending current work to multidimensional systems, to different communication channels, and to use different network resource indices.

\section{ACKNOWLEDGEMENTS}

The authors would like to thank Professor Geir E. Dullerud for his support and invaluable discussions in this research.

\section{REFERENCES}

[1] Äström, K.J., and B. Wittenmark, "Computer Controlled Systems Theory and Design", Prentice-Hall,1997.

[2] Bameih, B., and J.B. Pearson, "A General Framework for Linear Periodic Systems with Application to $\mathcal{H}_{\infty}$ Sampled-Date Control", IEEE Transactions on Automatic Control,37:418-435, 1992.

[3] Bameih, B., and J.B. Pearson, "The $\mathcal{H}_{2}$ Problems for Sampled-Data Systems", In Proc. IEEE 30th CDC, England, 1991.

[4] Chen, T., and B. A. Francis, "Optimal Sampled-Data Control Systems", Communications and Control Engineering Series, Springer, London, 1995.

[5] Delchamps, D. F., "Stabilizing a linear system with quantized state feedback", IEEE Transactions on Automatic Control,35:916C924, 1990.

[6] Elia, N., and S.K. Mitter, "Stabilization of Linear Systems With Limited Information", IEEE Transactions on Automatic Control,46(9):1384-1400, 2001.

[7] Fagnani, F., and S. Zampieri, "Stability analysis and synthesis for scalar linear systems with a quantized feedback", IEEE Transactions on Automatic Control,48(9):1569-1584, 2003.

[8] Fu, M., and L. Xie, "The Sector Bound Approach to Quantized Feedback Control", IEEE Transactions on Automatic Control, 50(11): 1698-1710, 2005.

[9] Gupta, V., A. F. Dana, R. M. Murray and B. Hassibi, "On the Effect of Quantization on Performance”, in Proc. 2006 IEEE ACC, 1364-1369, Minneapolis, 2006.

[10] Liberzon, D., "On Stabilization of Linear Systems with Limited Information", IEEE Transactions on Automatic Control,48(2):304307, 2003.

[11] M.-M. Ben Gaid, A. Çela, "Trading Quantization Precision for Sampling Rates in Networks Systems with Limited Communication", in Proc. 45th IEEE CDC, San Diego, 2005.

[12] Sha, L., X. Liu, M. Caccao, and G. Buttazzo, Online Control Optimization Using Load Driven Scheduling, in Proc. 39th IEEE CDC, Sydney, December, 2000.

[13] Nair, G.N., and R. J. Evans, "Exponential Stabilisability of finitedimensional linear systems with limited data rates", Automatica, 39:585-593, 2003.

[14] Seto, D., J. P. Lehoczky, L. Sha, and K. G. Shin, "On Task Schedulability in Real-Time Control System", in Proc. 17th IEEE Real-Time Systems Symposium, 13-21, 1996.

[15] Tatikonda, S., "Control Under Communication Constraints", Ph.D Thesis, M.I.T. 2000

[16] Tatikonda, S., and S. K. Mitter, "Control Under Communication Constraints", IEEE Transactions on Automatic Control, 49(7):10561068, 2004.

[17] Tatikonda, S., and S. K. Mitter, "Stochastic Linear Control Over a Communication Channel", IEEE Transactions on Automatic Control, 49(9):1549-1561, 2004.

[18] Verriest, E. and M. Egerstedt, Control with delayed and limited information: A first look, In Proceedings of the IEEE Conference on Decision and Control, 2002.

[19] Yuksel, S., and T. Basar, Optimal Signaling Policies for Decentralized Multi-Controller Stabilizability over Communication Channels, IEEE Transactions on Automatic Control, 52(10):1969-1974, 2007.

[20] Zhang, C., and G. Dullerud, Decentralized Control with Communication Bandwidth Limitation, In Proc. IEEE ACC 2007, NYC, 2007 